Meta

Journal des traducteurs

Translators' Journal

\title{
Regard rétrospectif sur la synergie entre traduction, lexicographie, terminologie et traductique
}

\section{Amélie Josselin-Leray, Aurélie Picton et Emmanuel Planas}

Volume 60, numéro 2, août 2015

$60^{\mathrm{e}}$ anniversaire. Les horizons de la traduction : retour vers le futur $60^{\text {th }}$ Anniversary. Translation's Horizons: Back to the Future

60mo aniversario. Los horizontes de la traducción: regreso al futuro

URI : https://id.erudit.org/iderudit/1032897ar

DOI : https://doi.org/10.7202/1032897ar

Aller au sommaire du numéro

Éditeur(s)

Les Presses de l’Université de Montréal

ISSN

0026-0452 (imprimé)

1492-1421 (numérique)

Découvrir la revue

Citer ce document

Josselin-Leray, A., Picton, A. \& Planas, E. (2015). Regard rétrospectif sur la synergie entre traduction, lexicographie, terminologie et traductique. Meta, 60(2), 346-346. https://doi.org/10.7202/1032897ar d'utilisation que vous pouvez consulter en ligne. 


\title{
Regard rétrospectif sur la synergie entre traduction, lexicographie, terminologie et traductique
}

\author{
Amélie Josselin-Leray \\ Université Jean Jaurès et CNRS, Toulouse, France \\ josselin@univ-tlse2.fr \\ Aurélie Picton \\ Université de Genève, Genève, Suisse \\ aurelie.picton@unige.ch \\ Emmanuel Planas \\ Université de Nantes et Université Catholique de l'Ouest, Angers, France \\ emmanuel.planas@univ-nantes.fr
}

En 60 ans, l'évolution de la traductologie n'aurait pu se faire sans celle conjointe de disciplines connexes qui lui sont aujourd'hui intimement liées et qui font de ce domaine ce qu'il devrait idéalement être: un domaine interdisciplinaire. On compte parmi ces disciplines la terminologie, la lexicographie et la traductique.

Nous souhaitons montrer à travers l'exemple du projet CRISTAL comment l'évolution de ces disciplines s'est mise en route depuis 60 ans pour permettre aujourd'hui leur rencontre et répondre à de nouveaux besoins de description et de réflexion en traduction. CRISTAL est un projet interdisciplinaire de 3 ans financé par l'Agence nationale pour la recherche (France); il fédère trois laboratoires et une entreprise langagière et vise à évaluer la génération automatisée et l'utilisation de contextes riches en connaissances (CRC) dans les outils de traductique. L'objectif principal de ce projet est l'amélioration de la qualité de la traduction via l'application de ses résultats aux outils classiques du traducteur spécialisé. Nous illustrerons la synergie entre ces disciplines, à travers la présentation d'une expérimentation auprès de traducteurs qui met en jeu: 1) l'évolution de ce qu'est une ressource pour le traducteur (en particulier les dictionnaires et la prise en compte du contexte dans ces outils) ; 2) l'évolution de l'environnement technique du traducteur, les outils dont il dispose, ainsi que l'évolution des techniques d'observation de l'acte de traduction; 3) l'évolution des méthodes et théories de la terminologie, son rapprochement avec l'ingénierie des connaissances et la linguistique de corpus, favorisant le développement du concept de CRC pour la traduction.

Cette synergie permet de faire émerger une vision renouvelée de la traductologie qui intègre maintenant les méthodes et les avancées théoriques de disciplines dont elle ne peut désormais plus être dissociée.

Aurélie Picton est professeure assistante en terminologie, lexicologie et technologies de la traduction. Amélie Josselin-Leray est Maître de conférences en linguistique anglaise et traduction. Emmanuel Planas est Maître de conférences en informatique pour la traduction. Ensemble, ils ont publié: Planas, E., Picton, A. et Josselin-Leray, A. (2014) : Exploring the Use and Usefulness of KRCs in Translation: Towards a Protocol - Terminology and Knowledge Engineering 2014, Berlin, 188-197. 